\title{
Salt effect on thermodynamics and kinetics of a single RNA base pair
}

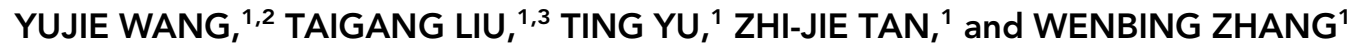 \\ ${ }^{1}$ Department of Physics and Key Laboratory of Artificial Micro and Nano-structures of Ministry of Education, School of Physics and Technology, \\ Wuhan University, Wuhan, Hubei, 430072, P.R. China \\ ${ }^{2}$ Department of Physics and Telecommunication Engineering, Zhoukou Normal University, Zhoukou, Henan, 466001, P.R. China \\ ${ }^{3}$ School of Medical Engineering, Xinxiang Medical University, Xinxiang, Henan, 453003, P.R. China
}

\begin{abstract}
Due to the polyanionic nature of RNAs, the structural folding of RNAs are sensitive to solution salt conditions, while there is still lack of a deep understanding of the salt effect on the thermodynamics and kinetics of RNAs at a single base-pair level. In this work, the thermodynamic and the kinetic parameters for the base-pair AU closing/opening at different salt concen-

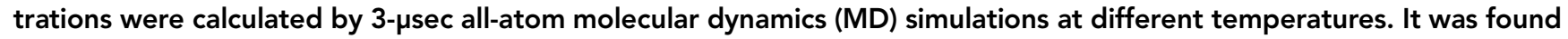
that for the base-pair formation, the enthalpy change $\Delta H$ is nearly independent of salt concentration, while the entropy change $\Delta S$ exhibits a linear dependence on the logarithm of salt concentration, verifying the empirical assumption based on thermodynamic experiments. Our analyses revealed that such salt concentration dependence of the entropy change mainly results from the dependence of ion translational entropy change for the base pair closing/opening on salt concentration. Furthermore, the closing rate increases with the increasing of salt concentration, while the opening rate is nearly independent of salt concentration. Additionally, our analyses revealed that the free energy surface for describing the basepair opening and closing dynamics becomes more rugged with the decrease of salt concentration.
\end{abstract}

Keywords: RNA base pair; salt effect; thermodynamics; kinetics

\section{INTRODUCTION}

RNAs perform critical cellular functions at the level of transcription, translation, and the expression and regulation of genetic information, where the functions are generally mediated through simple Watson-Crick base-pairing (Huynen et al. 1996; Rauscher et al. 1997; Bevilacqua and Blose 2008). Also, the closing and opening of base pairs is the fundamental process in RNA structure folding (Giudice et al. 2003; Sarkar et al. 2010; Nayak et al. 2012; Steinert et al. 2012; Wang et al. 2019a), consequently, a full understanding of the thermodynamics and kinetics of this process is critical to understanding the biological functions of RNAs.

Because RNAs are highly charged polyanionic molecules, the structure and conformational change of RNAs are sensitive to their ionic environments. The electrostatic interactions between metal ions and RNAs make a significant contribution to the thermodynamic stability of RNA secondary structures and their flexibility (Brion and Westhof 1997; Ippolito and Steitz 1998; Tinoco and

Corresponding authors: wbzhang@whu.edu.cn, zjtan@whu.edu.cn

Article is online at http://www.rnajournal.org/cgi/doi/10.1261/rna. 073882.119 .
Bustamante 1999; Heilman-Miller et al. 2001; Serra et al. 2002; Pyle 2002; Tan and Chen 2006, 2007, 2011; Draper 2008; Qiu et al. 2010, 2011; Kirmizialtin et al. 2012; Lipfert et al. 2014; Denesyuk and Thirumalai 2015; Drozdetski et al. 2016; Nakano et al. 2016; Sun et al. 2017; Fischer et al. 2018; Jin et al. 2018; Kolev et al. 2018; Chen and Pollack 2019). For instance, recent theoretical modeling (Tan and Chen 2006, 2007) on the salt-dependence of nucleic acid helix stability showed that nucleic acid helix stability can depend strongly on ion concentration, ion size and ion valence, and the ion-dependent loop stability made a significant contribution to the overall hairpin stability. Simultaneously, experimental measurements have found that the nucleic acid helix stability is affected by not only the sequence of helix but also apparently ion concentration (SantaLucia 1998; SantaLucia and Hicks 2004; Owczarzy et al. 2008; Chen and Znosko 2013). For example, thermodynamic and single-molecule measurements have both shown that an increase in salt concentration would

(C) 2020 Wang et al. This article is distributed exclusively by the RNA Society for the first 12 months after the full-issue publication date (see http://rnajournal.cshlp.org/site/misc/terms.xhtml). After 12 months, it is available under a Creative Commons License (Attribution-NonCommercial 4.0 International), as described at http:// creativecommons.org/licenses/by-nc/4.0/. 
apparently enhance the stability of secondary segments, and a logarithm salt-dependence of the folding free energy/entropy has been empirically derived at relatively high salt concentration (e.g., between $\sim 0.1-1$ M) (SantaLucia 1998; SantaLucia and Hicks 2004). In parallel, the influence of metal ions on the tertiary structure of RNA has been explored extensively (Woodson 2005; Draper et al. 2005; Koculi et al. 2007; Draper 2008; Tan and Chen 2011; Kim and Shapiro 2013; Li 2013; Wang et al. 2015; Wang and Xiao 2016; Shi et al. 2018; Xi et al. 2018; Hori et al. 2019; Jin et al. 2019). For example, Li (2013) studied the role of diffuse metal ions in stabilizing a 2-bp kissing complex by single-molecule techniques and found the significant contribution of diffuse cations to the stability of tertiary interaction.

Metal ions influence not only RNA structure stability but also the rate of conformational rearrangement and folding/unfolding pathways of RNAs (Bokinsky et al. 2003; Woodson 2005; Boerner et al. 2016; Vashishtha et al. 2016; Xu et al. 2016; Raper et al. 2018). For example, Bokinsky et al. (2003) probed the transition state for docking and undocking of the hairpin ribozyme using fluorescence resonance energy transfer (FRET) and found that the rate of docking increased with $\mathrm{Mg}^{2+}$ concentration, while the undocking rate remained constant. Recently, Shi et al. (2018) focused on the 3D structures and stability of RNA pseudoknots in monovalent and divalent ion solutions and found that the unfolding pathway of RNA pseudoknots can be significantly modulated by their sequences and solution ion conditions. However, these existing works mainly focused on the thermodynamics or kinetics of global RNA molecules and the salt effects on the closing and opening of single RNA base pair remain unclear.

Based on the extensive experimental data, the thermodynamic parameters of DNA and RNA base pairs at $1 \mathrm{M} \mathrm{Na}^{+}$ concentration have been derived empirically (SantaLucia 1998; Xia et al. 1998; SantaLucia and Hicks 2004). These parameters form the basis for the predictions of nucleic acid structures, thermodynamics, and folding kinetics (Serra and Turner 1995; Zhang and Chen 2002; SantaLucia and Hicks 2004; Zhao et al. 2010). Moreover, some salt extensions of the thermodynamic parameters of DNAs and RNAs have been derived either by experiments (Blake and Delcourt 1998; SantaLucia 1998; SantaLucia and Hicks 2004; Owczarzy et al. 2004, 2008) or through theoretical modeling (Tan and Chen 2006, 2007). However, the iondependent kinetic parameters, such as the opening and closing rates of a single base pair, are still lacking until now. RNA folding kinetics is directly tied to RNA biological functions. The functions of ribozymes (Bartel and Szostak 1993), anti-HIV RNA aptamers (Ellington and Szostak 1990) and gene expression regulators, such as miRNA, siRNA, and riboswitches (Bartel 2004; Nudler and Mironov 2004), are often kinetically controlled. Experiments have demonstrated that helix formation is a zipping process
(Kuznetsov and Ansari 2012), and unraveling the kinetics of a single base pair is crucial to understand the overall kinetics of the full length RNA. Furthermore, the mechanism of how salt ions influence the opening and closing rate of a single base pair remains unclear. Therefore, understanding the salt effect on thermodynamics and kinetics of a single RNA base pair is important for understanding the biological functions of RNAs.

Recently, the thermodynamic and kinetic parameters of the AU base pair at high salt have been derived by 3- $\mu$ sec all-atom MD simulations at different temperatures (Wang et al. 2016); and furthermore, the effects of nearest neighbor and next nearest neighbor on the thermodynamic and kinetic properties have been explored (Wang et al. 2018).

In this work, the salt effects on the thermodynamic and kinetic parameters of the AU base pair were examined by our 3- $\mu \mathrm{sec}$ all-atom MD simulations. Our calculations suggested that for the opening/closing of a base pair, the enthalpy change $\Delta H$ is almost unaffected by ion concentration, while the entropy change $\Delta S$ is strongly dependent on ion concentration. Our analyses revealed that such salt dependence of entropy change is mainly attributed to the change of ion translational entropy due to base-pair opening/closing. Furthermore, with the increase of ion concentration, the closing rate increases while the opening rate remains nearly invariant. In addition, the free energy surface for the opening and closing dynamics of the base pair was found to become more rugged with the decrease of salt concentration.

\section{RESULTS AND DISCUSSION}

\section{Determination of closed state (cs), open state (os), and transition state (ts)}

Similarly to our previous analyses (Wang et al. 2016, 2018), the terminal base-pair $\mathrm{AU}$ at different $\mathrm{NaCl}$ concentration would undergo the two-state closing-opening switch process (see Fig. 1) through a transition state. The corresponding structures can be divided into closed state, open state and transition state according to the time-dependent RMSD (root mean square deviation) of the two terminal nucleotides $A$ and $U$ relative to the initial structure, and the torsional angle $\zeta$, which is the dihedral angle formed by the four atoms $\mathrm{C}^{\prime}(i)-\mathrm{O} 3^{\prime}(i)-\mathrm{P}(i+1)-\mathrm{O}^{\prime}(i+1)$ (Hershkovitz et al. 2003), where i represents the $i$-th nucleotide in a polynucleotide.

For the closed state, the RMSD is $\sim 0.7 \AA$ and $\zeta$ is centered in the region around $\sim-75^{\circ}\left(-50^{\circ}\right.$ to $\left.-100^{\circ}\right)$, as shown in Figure 2. The corresponding conformations are that the terminal two nucleotides vibrate slightly only around their starting positions, and the interactions of terminal basepairing and base stacking are not disrupted. As A-RNA helix has a narrow deep groove and higher negative charge density, $\mathrm{Na}^{+}$ions are mainly concentrated near the 


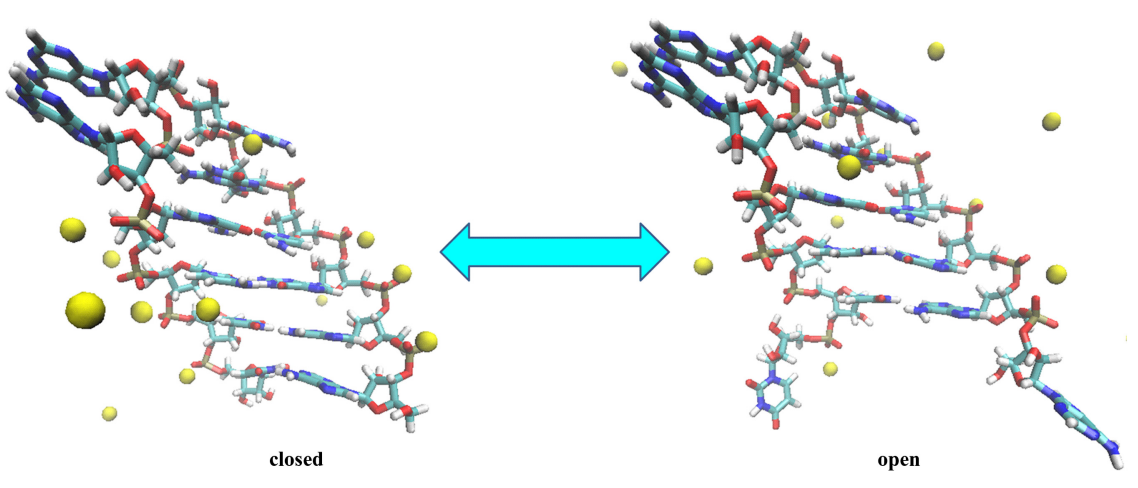

FIGURE 1. The illustration for the simulation system in the work, where an RNA hairpin is in salt solution. In the simulations, the hairpin (PDB code, 1ZIH) was fixed with strong harmonic constraint except for the two free terminal nucleotides. The left and right panels show the two typical states of the hairpin with the closed and open terminal base pair: left, closed state; right, open state. The three-dimensional structures are shown with VMD software (Humphrey et al. 1996), and the brown balls denote monovalent sodium ions.

negatively charged phosphate group and in the deep groove binding to the N7 atom of terminal nucleotide A.

For the open state, the RMSD is larger than $2 \AA$ and $\zeta$ is centered in the region around $\sim 50^{\circ}\left(30^{\circ}-100^{\circ}\right)$, as shown in Figure 2. The corresponding conformations are that the two terminal nucleotides flip out into the solvent, and the interactions of terminal base-pairing and base-stacking are both disrupted, some of the $\mathrm{Na}^{+}$ions in the deep groove move out. However, there are still some $\mathrm{Na}^{+}$ions binding to the phosphate group.

For the transition state, the RMSD values fluctuate beyond $2 \AA$ with a very short residence time; however, the torsion angle is still in the region of closed state, and the bases are flipped out into the solvent; see Figure 3. It was found that all the transition paths from the closed to open states or from the open to closed state go through the transition state. The actual transition paths from the closed to open state and from the open to closed state take roughly the same amount of time as the residence time of an oto and ctc configuration, respectively. According to the location of the transition state that appeared, the transition states (Dokholyan et al. 2000; Zhang et al. 2006) can be divided into ctc, which transits from the closed state and subsequently returns to the closed state

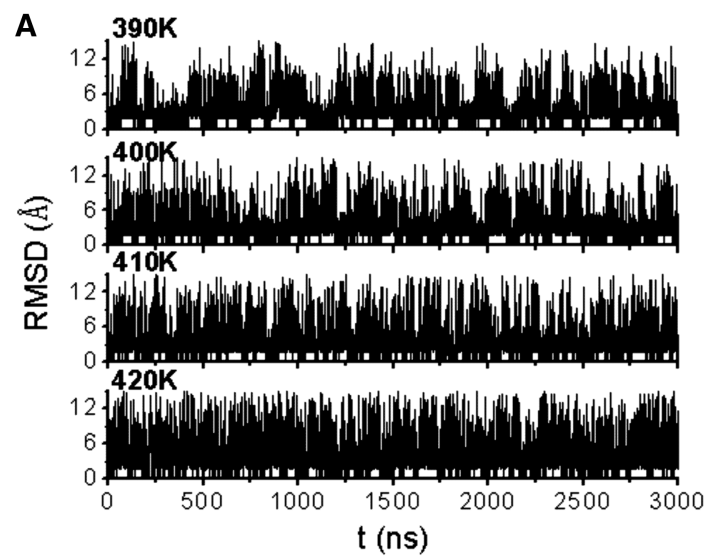

C

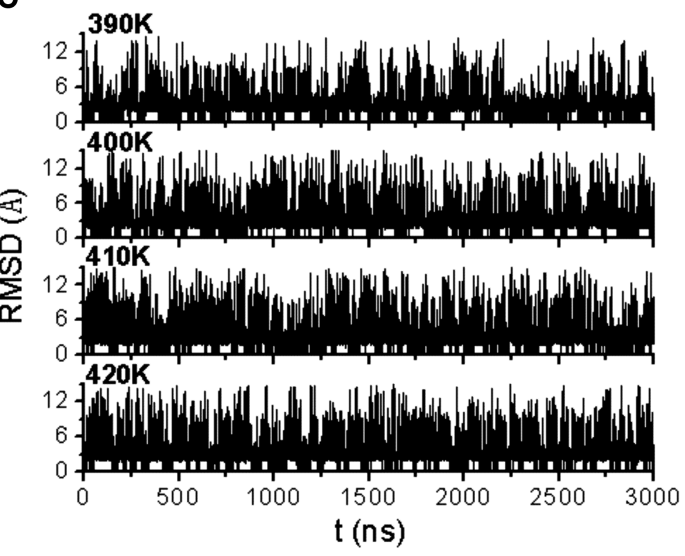

B

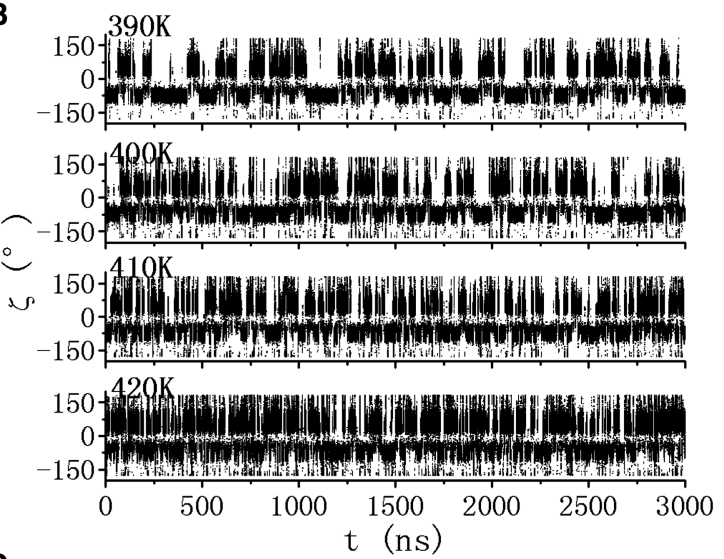

D

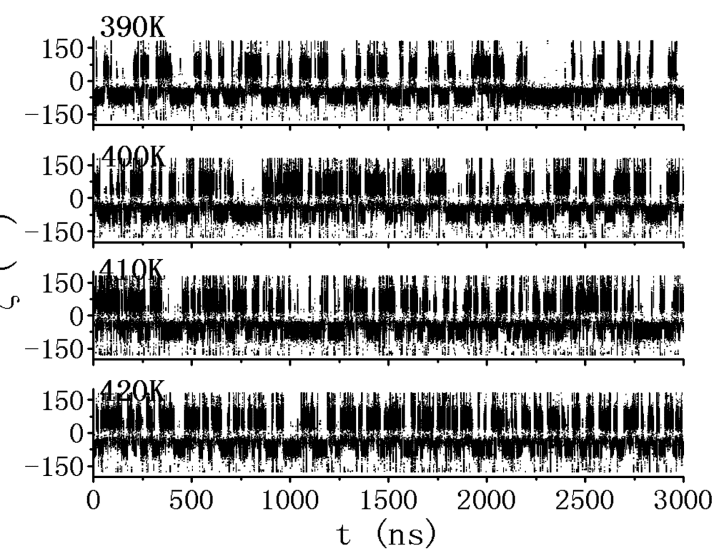

FIGURE 2. The RMSD values and torsional angles $\zeta$ for the AU base pair at different ion concentrations during the whole simulation times (0-3000 nsec): $(A, B) 0.1 \mathrm{M} \mathrm{NaCl}$ at $390 \mathrm{~K}, 400 \mathrm{~K}, 410 \mathrm{~K}$, and $420 \mathrm{~K} ;(C, D) 0.05 \mathrm{M} \mathrm{NaCl}$ at $390 \mathrm{~K}, 400 \mathrm{~K}, 410 \mathrm{~K}$, and $420 \mathrm{~K}$. 

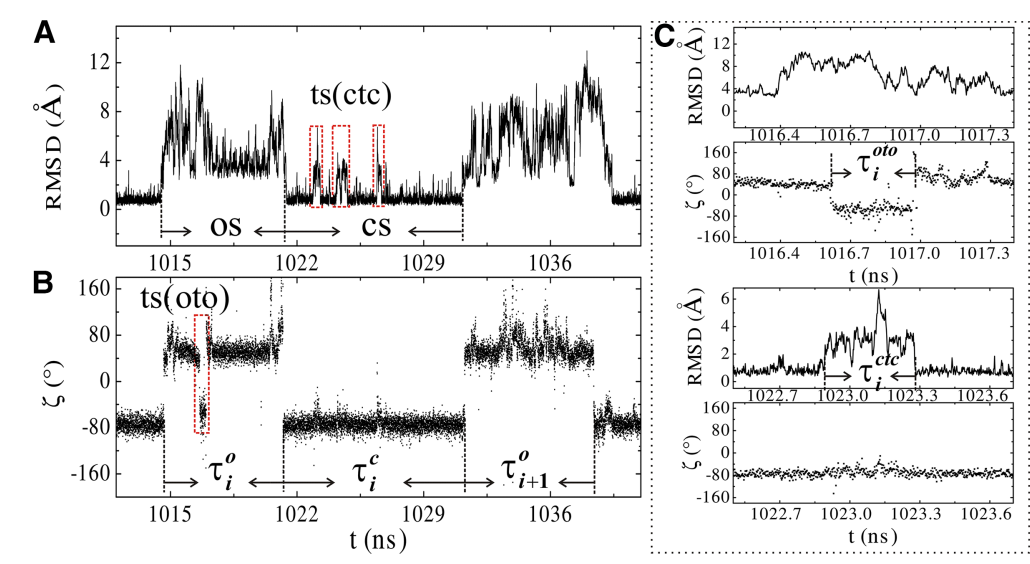

FIGURE 3. (A) The RMSD values and (B) torsional angles $\zeta$ for base-pair $A U$ at $0.1 \mathrm{M} \mathrm{NaCl}$ among the simulation times of 1012-1041 nsec at $420 \mathrm{~K}$. (C) The RMSD and torsional angles $\zeta$ near the transition states.

(see Fig. 3A,C), and oto, which transits from the open state and subsequently returns to the open state (see Fig. 3B,C). The difference of transition states ctc and oto is nominal; the structures of the two states are similar. But the transition rates from the transition states to the open state and closed state are different, so the lifetimes of the ctc and oto are different. When the conformations are in the transition states, since the twist angle does not flip, some $\mathrm{Na}^{+}$ions can still be trapped around the released negatively charged phosphate group. However, the flipped bases of two terminal nucleotides $A$ and $U$ swing constantly in solvent, sometimes individual $\mathrm{Na}^{+}$ion binds to the $\mathrm{O} 4$ atom of terminal nucleotide $U$, but the time is relatively short.

\section{The thermodynamic parameters of the terminal $A U$ base pair at different ion concentrations}

The population distribution of the closed, open and transition states at a given temperature can be calculated as:

$$
\begin{aligned}
& p_{c}=\sum_{i=1}^{N_{c}} \tau_{i}^{c} / \tau_{,} \\
& p_{o}=\sum_{i=1}^{N_{o}} \tau_{i}^{o} / \tau_{,} \\
& p_{t}=\sum_{i=1}^{N_{t}} \tau_{i}^{t} / \tau_{,}
\end{aligned}
$$

where $\tau$ is the total simulation time, $\tau_{i}^{c}, \tau_{i}^{o}$, and $\tau_{i}^{t}$ are the $i$-th dwelling period of the conformations in the closed, open, and transition states, respectively, and $N_{c}, N_{o}$, and $N_{t}$ are the total number of snapshots of those conformations in the closed, open and transition states, respectively. As shown in Figure $4 A, B$, when the simulation time exceeds $\sim 2 \mu \mathrm{sec}$, the occupied probability of the closed state approximately reaches a stable value. Therefore, the simulation times were all set to be $3000 \mathrm{nsec}$ at each temperature to ensure that all the simulations can remain in the closing-opening two-state equilibrium for enough time. The probabilities of the closed, open and transition states at different temperatures are listed in Table 1.

As the probabilities of the transition states are much smaller than those of the closed and open states, we would first treat the conformations as the (closed and open) two-state model. According to the equilibrium population distribution, the free energy difference $\Delta G$ between the two states (closed and open state) for the AU base pair can be calculated through:

$$
\Delta G=-k_{B} T \ln \left(\frac{p_{0}}{p_{c}}\right)
$$

where $k_{B}$ is the Boltzmann constant, $T$ is the absolute temperature, and $p_{o}$ and $p_{c}$ are the occupied populations of the open and closed states, respectively.

As shown in Figure $4 C$, the free energy difference $\Delta G$ and the temperature $T$ have a linear relationship. According to $\Delta G=\Delta H-T \Delta S$, where $\Delta H$ and $\Delta S$ are the enthalpy and entropy changes for the transition between the closed and open states, the thermodynamic parameters of the terminal AU base pair are found to be $\Delta H=-7.31 \mathrm{kcal} /$ $\mathrm{mol}$ and $\Delta S=-18.86$ eu at $0.1 \mathrm{M} \mathrm{NaCl}$, and $\Delta H=-7.30$ $\mathrm{kcal} / \mathrm{mol}$ and $\Delta S=-18.98 \mathrm{eu}$ at $0.05 \mathrm{M} \mathrm{NaCl}$. At $0.5 \mathrm{M}$ $\mathrm{NaCl}$, the thermodynamic parameters of terminal basepair $A U$ are $\Delta H=-7.30 \mathrm{kcal} / \mathrm{mol}$ and $\Delta S=-18.50 \mathrm{eu}$ (Wang et al. 2016). As shown in Figure 4D, it can be seen that the enthalpy changes $\Delta H$ of the base-pair $A U$ remain nearly invariant for $0.5,0.1$, and $0.05 \mathrm{M} \mathrm{NaCl}$, and the difference of $\Delta H$ for different $\left[\mathrm{Na}^{+}\right]$is very small $(<\sim 0.01 \mathrm{kcal} / \mathrm{mol})$. However, the entropy changes $\Delta S$ are apparently different for $0.5,0.1$, and $0.05 \mathrm{M} \mathrm{NaCl}$, and the entropy change gradually decreases with the increase of salt concentration in a linear logarithm-dependence. It is encouraging that such salt-dependence of enthalpy and entropy changes is in good accordance with the assumption made in the experimental and theoretical salt-extensions of thermodynamic parameters for DNA/RNA basepair closing/opening in the salt range of [0.05-1 M] (SantaLucia 1998; SantaLucia and Hicks 2004; Tan and Chen 2006, 2007; Owczarzy et al. 2008; Chen and Znosko 2013). In the following, we examine the contribution of ion entropy to the linear logarithm-dependence of entropy change $\Delta S$ on salt.

According to the counterion condensation theory (Manning 1978, 2001, 2007; Lin et al. 2019), the ion condensation entropy change for a base-pair closing (i.e., 

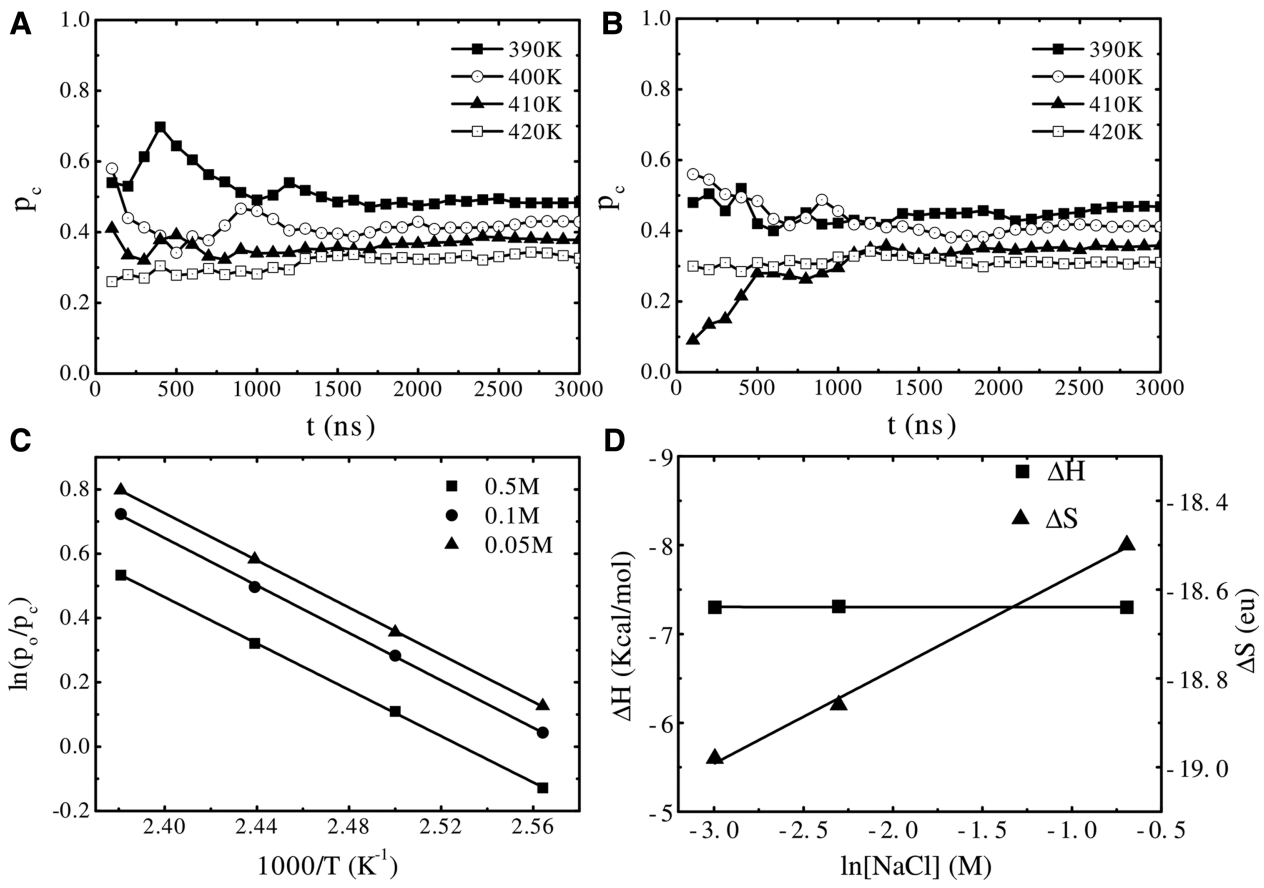

FIGURE 4. The occupied population of the closed state versus the simulation time at (A) $0.1 \mathrm{M} \mathrm{NaCl}$, and (B) $0.05 \mathrm{M} \mathrm{NaCl}$. (C) The temperature dependence of $\ln \left(p_{o} / p_{c}\right)$. (Symbols) Full square for $0.5 \mathrm{M} \mathrm{NaCl}$; full circle for $0.1 \mathrm{M} \mathrm{NaCl}$; full triangle for $0.05 \mathrm{M} \mathrm{NaCl}$ : from MD simulations; (lines) from linear fitting. (D) The $\mathrm{NaCl}$ concentration dependence of $\Delta H$ and $\Delta S$. (Symbols) Full square for $\Delta H$; full triangle for $\Delta S$ : from MD simulations; (lines) from linear fitting.

open state $\rightarrow$ closed state) can be given by

$$
\Delta S_{i o n}\left(\mathrm{Na}^{+}\right)=-R\left[N_{b}^{c} \ln \left(\frac{C_{b}^{c}}{C_{0}}\right)-N_{b}^{o} \ln \left(\frac{C_{b}^{o}}{C_{0}}\right)\right],
$$

where $R$ is the gas constant $(R=1.987 \mathrm{cal} / \mathrm{K} / \mathrm{mol}), N_{b}^{c}$ and $N_{b}^{o}$ are the numbers of $\mathrm{Na}^{+}$binding to the RNA when the terminal base-pair $A U$ is in the closed (c) state and open (o) state, respectively. $C_{b}^{c}$ and $C_{b}^{\circ}$ are the corresponding concentration of binding ions, respectively. $C_{0}$ is the bulk salt concentration.

According to the ion concentration distributions around the phosphorus atoms shown in Figure 5A, we calculated the numbers of binding $\mathrm{Na}^{+}$for the RNA in the closed and open states of the terminal base pair. Our calculations show that the changes in the average number of binding ions due to the formation of the terminal base pair are approximately invariant for $0.5,0.1$, and $0.05 \mathrm{M} \mathrm{NaCl}$. Generally, in thermodynamic measurements and modeling, $1 \mathrm{M} \mathrm{Na}^{+}$is used as the standard ionic condition, and thus, the salt-extension of the entropy change due to the formation of the terminal base pair can be approximately expressed as based on Equation 3:

$$
\Delta S_{\text {ion }}\left(\left[\mathrm{Na}^{+}\right]\right)=\Delta S_{\text {ion }}(1 \mathrm{M})+R\left(N_{b}^{c}-N_{b}^{o}\right) \ln \left(\left[\mathrm{Na}^{+}\right]\right),
$$

where the difference in $\left[N_{b}^{c} \ln \left(C_{b}^{c}\right)-N_{b}^{o} \ln \left(C_{b}^{\circ}\right)\right]$ from $1 \mathrm{M}$ $\mathrm{Na}^{+}$is ignored due to the relatively small value (e.g., for

TABLE 1. The average lifetime $\tau_{\text {ave }}(\mathrm{ns})$, the occupied probability $p$, and the total number $N$ of occurrences of conformations at closed,

\begin{tabular}{|c|c|c|c|c|c|c|c|c|c|c|c|c|c|}
\hline \multirow{2}{*}{$\begin{array}{l}\text { Concentration } \\
\mathrm{Na}^{+}(\mathrm{M})\end{array}$} & \multirow{2}{*}{$\begin{array}{c}\text { Temperature } \\
T(\mathrm{~K})\end{array}$} & \multicolumn{3}{|c|}{ Closed state (cs) } & \multicolumn{3}{|c|}{ Open state (os) } & \multicolumn{3}{|c|}{ Transition state (ctc) } & \multicolumn{3}{|c|}{ Transition state (oto) } \\
\hline & & $\tau_{\text {ave }}^{c}$ & $N_{c}$ & $p_{c}$ & $\tau_{\text {ave }}^{o}$ & $N_{\circ}$ & po & $\tau_{\text {ave }}^{\text {ctc }}$ & $N_{c t c}$ & $p_{c t c}$ & $\tau_{\text {ave }}^{\text {oto }}$ & $N_{\text {oto }}$ & poto \\
\hline \multirow[t]{4}{*}{0.1} & 390 & 26.67 & 55 & 0.49 & 28.39 & 54 & 0.51 & 0.299 & 314 & 0.031 & 0.296 & 114 & 0.011 \\
\hline & 400 & 20.46 & 63 & 0.43 & 27.59 & 62 & 0.57 & 0.289 & 336 & 0.032 & 0.290 & 158 & 0.015 \\
\hline & 410 & 16.21 & 70 & 0.38 & 27.03 & 69 & 0.62 & 0.285 & 410 & 0.038 & 0.284 & 188 & 0.017 \\
\hline & 420 & 12.89 & 76 & 0.33 & 26.58 & 76 & 0.67 & 0.281 & 357 & 0.033 & 0.282 & 221 & 0.020 \\
\hline \multirow[t]{4}{*}{0.05} & 390 & 27.01 & 52 & 0.47 & 31.27 & 51 & 0.53 & 0.301 & 346 & 0.034 & 0.299 & 127 & 0.012 \\
\hline & 400 & 20.95 & 59 & 0.41 & 30.41 & 58 & 0.59 & 0.291 & 364 & 0.035 & 0.289 & 176 & 0.016 \\
\hline & 410 & 16.54 & 65 & 0.36 & 29.61 & 65 & 0.64 & 0.284 & 459 & 0.043 & 0.285 & 204 & 0.019 \\
\hline & 420 & 12.94 & 72 & 0.31 & 29.13 & 71 & 0.69 & 0.280 & 383 & 0.035 & 0.282 & 245 & 0.023 \\
\hline
\end{tabular}
open and transition states for base-pair $\mathrm{AU}$ at different $\mathrm{NaCl}$ concentrations and temperatures from the 3- $\mu$ sec simulations 

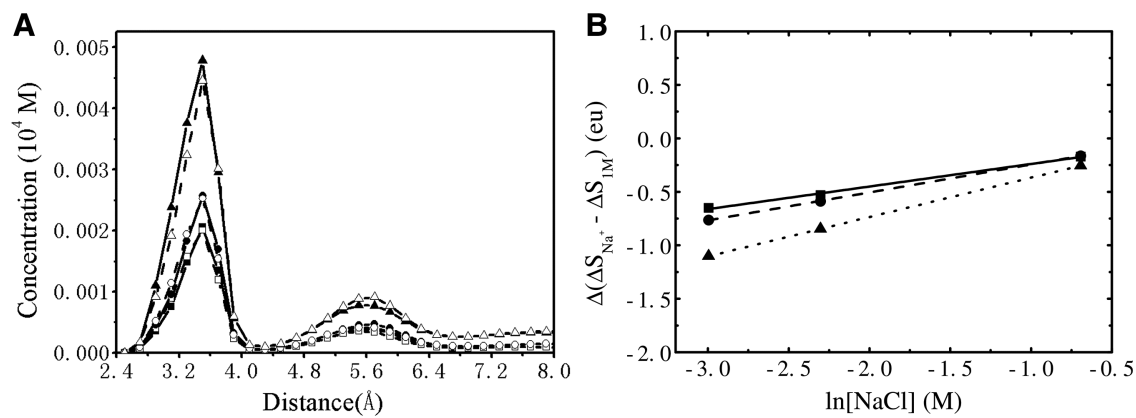

FIGURE 5. (A) The average concentration of $\mathrm{Na}^{+}$around phosphorus atoms of terminal base pair over the conformations of the closed state (full symbols) and open state (open symbols) for $0.5,0.1$, and $0.05 \mathrm{M} \mathrm{NaCl}$ (from top to bottom). Here, $T=410 \mathrm{~K}$. (Symbol) Triangle, $0.5 \mathrm{M}$; circle, $0.1 \mathrm{M}$; square, $0.05 \mathrm{M}$. (B) The ion concentration dependence of $\Delta=\Delta S_{\mathrm{Na}^{+}}-\Delta S_{1 \mathrm{M}}$. (Symbol) Square, from Equation 2; circle, from Equation 4; triangle, from the empirical formula of SantaLucia et al. (SantaLucia 1998).

$0.1 \mathrm{M} \mathrm{Na}^{+}$, such difference is $\sim 0.047 \mathrm{eu}$, while the second term in Equation 4 is $\sim 0.59 \mathrm{eu}$ ). We would like to compare the slope of the linear logarithm-dependence in Equation 4 based on the information of ion binding from the MD data, with that in the empirical formula from SantaLucia (1998). As shown in Figure 5B, the slope of the logarithm-dependence in Equation 4 is $\sim 0.26$, a close value to that $(\sim 0.21)$ from the above thermodynamic calculations. However, such slope of the dependence $(\sim 0.26)$ is slightly smaller than that $(\sim 0.368)$ from the empirical salt-extension from SantaLucia (1998). Such deviation between our calculations and the empirical formula may be partially attributed to the end effect and the small RNA employed in the MD simulations, because the formation of a base pair at ends or a base pair in a smaller RNA would generally cause a smaller increase in the number of binding ions. Thus, our analyses also suggest that for a basepair formation, ion translational entropy may make the major contribution to the logarithm-dependence of entropy change on salt concentration.

The formation of a base pair from the open state results in entropy reduction, which comes from the RNA bases' entropy loss due to the conformation restriction for the pairing, the ion's entropy loss due to the binding with the RNA base pair and the solvent's entropy loss due to hydration. As the salt concentration increases, the entropy loss of ion binding and hydration will decrease. Our analyses revealed that such salt concentration dependence of the entropy change mainly results from the dependence of ion translational entropy change for the base-pair closing/opening on salt concentration. As our calculated results are slightly transition states. smaller than that from the empirical salt-extension, it is likely that the hydration effects on the terminal base pair are less than that in the groove. The enthalpy change and the opening barrier, which mainly comes from the stacking interaction and the hydrogen bond between the base pairs, may not be affected by the salt concentration.

\section{The kinetic parameters of the terminal $A U$ base pair at different ion concentrations}

The average lifetimes $\tau_{\text {ave }}$ of the closed, open and transition states were calculated through $\tau_{a v e}=\sum_{i=1}^{N} \tau_{i} / N$, where $N$ is the total number of times the base pair resides in each state and $\tau_{i}$ is the $i$-th lifetime of the conformation in the corresponding state. The average times of the states are all listed in Table 1. It can be seen that the average lifetime of the closed and open states is much longer than those of the

For the closing-opening two-state model (Wang et al. 2016 , 2018), the closing rate from the open state to the closed state $k_{+}$and the opening rate from the closed state to the open state $k_{-}$can be calculated using the formulas $k_{+}=1 / \tau_{\text {ave }}^{o}$ and $k_{-}=1 / \tau_{\text {ave }}^{c}$, where $\tau_{\text {ave }}^{o}$ is the average lifetime of the open state and $\tau_{\text {ave }}^{c}$ is the average time of the closed state. Figure 6A shows the temperature dependence of the closing rate and the opening rate at $0.5,0.1$, and $0.05 \mathrm{M} \mathrm{NaCl}$. It can be seen that the opening rate at $0.5,0.1$, and $0.05 \mathrm{M} \mathrm{NaCl}$ exhibits a strong dependence on temperature, whereas the closing rate shows only weak temperature-dependent behavior. Furthermore, the opening rate is invisibly affected by ion
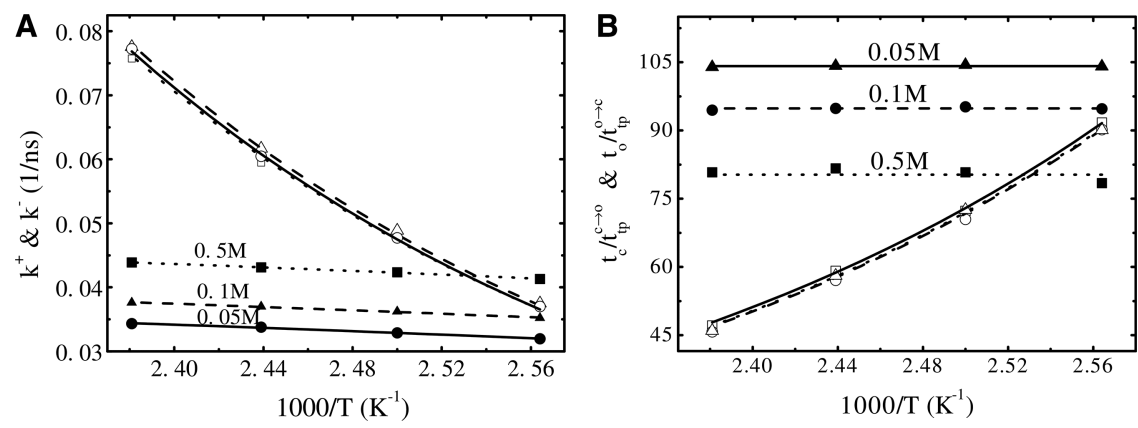

FIGURE 6. (A) Temperature dependence of the closing rate $k_{+}$(full symbols) and the opening rate $k_{-}$(open symbols) at different ion concentrations. (B) Temperature dependence of the ratios of $t_{c} / t_{t p}^{c \rightarrow 0}$ (open symbols) and $t_{0} / t_{t p}^{o \rightarrow c}$ (full symbols) at different ion concentrations. (Symbols) From the MD simulations (square for $0.5 \mathrm{M} \mathrm{NaCl}$; circle for $0.1 \mathrm{M} \mathrm{NaCl}$; triangle for $0.05 \mathrm{M} \mathrm{NaCl}$ ); (Lines) Fitted with Equations 5 and 6. 
concentration, while the closing rate is apparently dependent on ion concentration. The barrier for the formation of a single base pair from the open state is the reduction in entropy, which results from the RNA bases' entropy loss due to the conformation restriction for the pairing with each other, the ion's entropy loss due to the binding with the RNA base pair and the solvent's entropy loss due to hydration. As the salt concentration increases, the entropy loss of ion binding and hydration will decrease, and then, at a given temperature, the closing rate increases visibly as ion concentration is increased.

Based on the three-state (closed, open and transition state) model, the transition rates from the transition state to the closed state $k_{t \rightarrow c}$ and to the open state $k_{t \rightarrow 0}$ can be calculated as $k_{t \rightarrow c}=1 / \tau_{\text {ave }}^{c t c}$ and $k_{t \rightarrow o}=1 / \tau_{\text {ave }}^{\text {oto }}$ where $\tau_{a v e}^{c t c}$ is the average lifetime of transition state ctc and $\tau_{\text {ave }}^{\text {oto }}$ is the average lifetime of the transition state oto. The transition path time $t_{t p}^{c \rightarrow 0}$ from the closed state to the open state and that from the open state to the closed state $t_{t p}^{o \rightarrow c}$ are: $t_{t p}^{c \rightarrow o}=\tau_{\text {ave }}^{o t o}$ and $t_{t p}^{o \rightarrow c}=\tau_{\text {ave }}^{c t c}$, respectively. According to the transition-state theory (Bruce et al. 1988; Hänggi et al. 1990; Hummer 2004; Chung et al. 2009; Chung and Eaton 2013):

$$
\begin{gathered}
t_{c}=\frac{1}{k_{-}}=\frac{2 \pi}{\beta D^{*} \omega^{*} \omega_{c}} \exp \left(\beta \Delta G_{c}\right), \\
t_{0}=\frac{1}{k_{+}}=\frac{2 \pi}{\beta D^{*} \omega^{*} \omega_{o}} \exp \left(\beta \Delta G_{o}\right), \\
t_{t p}^{o \rightarrow c}=\frac{1}{\beta D^{*}\left(\omega^{*}\right)^{2}} \ln \left(2 e^{\gamma} \beta \Delta G_{o}\right), \\
t_{t p}^{c \rightarrow o}=\frac{1}{\beta D^{*}\left(\omega^{*}\right)^{2}} \ln \left(2 e^{\gamma} \beta \Delta G_{c}\right),
\end{gathered}
$$

where $\beta=1 / k_{B} T, k_{B}$ is the Boltzmann constant, $T$ is the absolute temperature, $D^{*}$ is the diffusion coefficient at the free energy barrier top, $\left(\omega^{*}\right)^{2},\left(\omega_{c}\right)^{2}$ and $\left(\omega_{\circ}\right)^{2}$ are the curvatures of the free energy surface at the barrier top and the closed and open states, respectively. $\gamma$ is the Euler's constant, and $\Delta G_{c}$ and $\Delta G_{0}$ are the free energy barrier heights of the closed and open states, respectively.

According to Equations 5 and 6 , the ratios of $t_{c} / t_{t p}^{c \rightarrow 0}$ and $t_{0} / t_{\text {tp }}^{\mathrm{O} \rightarrow \mathrm{c}}$ depend only on the free energy barrier, $\omega^{*} / \omega_{c}$ and $\omega^{*} / \omega_{0}$, but are independent of $D^{*}$. As shown in Figure 6B, $t_{c} / t_{t p}^{c \rightarrow 0}$ shows a strong temperature-dependence, whereas $t_{0} / t_{\text {tp }}^{o \rightarrow c}$ is nearly independent of temperature. Furthermore, $t_{c} / t_{t p}^{c \rightarrow 0}$ is invisibly affected by ion concentration, while $t_{0} / t_{t p}^{o \rightarrow c}$ is strongly dependent on ion concentration. Considering that $\omega^{*} / \omega_{0}$ is a constant and was generally defined as a constant in modeling protein folding kinetics (Chung and Eaton 2013), the free energy barrier of the base pair from the open state to the closed state should be proportional to temperature $T, \Delta G_{0} \propto T$. The free energy changes of the closed and open states were: $\Delta G=$ $\Delta G_{c}-\Delta G_{0}=\Delta H-T \Delta S$. By fitting the curves, the free energy barriers from the closed state to the open state for the terminal AU base pair at $0.1 \mathrm{M}$ and $0.05 \mathrm{M} \mathrm{NaCl}$ are equal to $\sim-7.31 \mathrm{kcal} / \mathrm{mol}$, which corresponds to the enthalpy changes $\Delta H$ between the closed state and open state. The free energy barrier from the open state to the closed state is $\Delta G_{0}=T \Delta S$, where $\Delta S$ is the entropy change between the open and closed states.

It has been suggested that the diffusion coefficient $D^{*}$ should exhibit super-Arrhenius behavior for a diffusion in a rough energy landscape (Bryngelson and Wolynes 1989), that is, $D^{*} \propto \exp \left[-\left(\Delta E / k_{B} T\right)^{2}\right]$, where $(\Delta E)^{2}$ is the local mean-squared fluctuation in energy and is a measure of the underlying landscape roughness. Afterwards, according to $\Delta G_{0}=T \Delta S, \Delta G_{c}=\Delta H$, and Equation $5, \Delta E$ is found to be $0.78 \mathrm{kcal} / \mathrm{mol}$ and $0.80 \mathrm{kcal} / \mathrm{mol}$ for the $A U$ base pair at $0.1 \mathrm{M} \mathrm{NaCl}$ and $0.05 \mathrm{NaCl}$ by fitting the temperature-dependent opening and closing times, respectively. Additionally, $\Delta E$ for the $A U$ base pair at $0.5 \mathrm{M} \mathrm{NaCl}$ is $0.76 \mathrm{kcal} / \mathrm{mol}$ (Wang et al. 2016). Therefore, the analyses indicate that the free energy landscape of the terminal base-pair AU becomes more rugged as salt concentration decreases, that is, local mean-squared fluctuation becomes more apparent.

\section{Conclusions}

In summary, the thermodynamic and kinetic parameters of the AU base pair at different ion concentrations were derived by 3 - $\mu$ sec all-atom molecular dynamics (MD) simulations at different temperatures. Our calculations showed that the enthalpy change $\Delta H$ for the closing/opening of a base pair is nearly unaffected by ion concentration, whereas the entropy change $\Delta S$ is strongly dependent on ion concentration, which indicated that $\mathrm{Na}^{+}$binding to the RNA group can promote the folding of an open base pair by reducing the disorder of the unfolded conformations. The ion concentration dependence of the entropy change mainly results from the differences of ion translational entropy changes for the closing/opening of the base pair at different ion concentrations. The closing and opening rates show different temperature-dependent behaviors. The closing rate is greatly affected by salt concentration and the closing rate increases with the increase of salt concentration, whereas the opening rate is almost unaffected by salt. Our analyses show that the free energy landscape of the AU base pair becomes rougher as ion concentration is decreased.

From the above, our molecular dynamic simulations provide an atomistic-level picture for the closing-opening transition of base-pair $\mathrm{AU}$, which is difficult to be observed experimentally, and reveal the microscopic mechanism for the effects of ions on thermodynamic and kinetic parameters of a single RNA base pair. Opening the terminal base pair would disrupt the stacking interactions of the terminal base pair with the next base pair, which would increase the probability of the next base pair to be destabilized. 
Although the accuracies of the simulation-based calculations and analyses may be limited by the force field and simulation time, the all-atom MD simulation can be still an effective tool for exploring more complex kinetic processes, such as RNA pseudoknot unfolding kinetics (Zhang et al. 2011), the effect of argonaute protein on miRNA's seed base (Wang et al. 2019b), and catalytic interactions between ions and RNA (Casalino and Magistrato 2016). A very recent work has shown that an MD-based treatment with the combination of other methods such as master equation and kinetic Monte Carlo technique is effective for analyzing RNA helix-terminal basepairing (Wang et al 2019a). Nevertheless, the present work provides a microscopic understanding on the salt dependence of RNA thermodynamics and kinetics at single base-pair level.

\section{MATERIALS AND METHODS}

Following our preceding works (Wang et al. 2016, 2018), the initial structure of sequence $5^{\prime}-$ (AAGCGCAAGCUU) -3 ', which includes four base pairs and a tetraloop, was obtained from the crystal structure (PDB ID:1ZIH). In our simulations, the model molecule was solvated in a triclinic box with TIP3P (Jorgensen et al. 1983; Mahoney and Jorgensen 2000) water molecules as solvent. The counterions of $\mathrm{Na}^{+}$and the salt of $\mathrm{NaCl}$ were added to ensure that our simulated systems are fully neutralized.

Our MD simulations were carried out using the GROMACS 4.5.6 simulation package (Hess et al. 2008) with the Amber10 all-atom force field (Wang and Kollman 2001; Wang et al. 2000; Pérez et al. 2007; Zgarbová et al. 2011), and the periodic boundary condition was employed in the simulations. The velocity rescaling (Bussi et al. 2007) and the Parrinello-Rahman barostat algorithm (Parrinello and Rahman 1981) were used to achieve constant temperature and pressure, respectively. The ParticleMesh Ewald method (Darden et al. 1993; Essmann et al. 1995) was used to treat long-range interactions with a $10 \AA$ cutoff, and Lennard-Jones interactions (Lennard-Jones 1931; Dzyaloshinskii et al. 1961) were truncated at $10 \AA$. The bond lengths of the solute were constrained by the LINCS algorithm (Hess et al. 1997), and the geometry of water molecules was kept completely rigid with the SETTLE algorithm (Miyamoto and Kollman 1992). The neighboring grid search method (Hess et al. 2008), which was updated every ten steps, was also employed. In the simulations, a time step of $2 \mathrm{fsec}$ was used in the calculations and the coordinates of atoms were saved every 2 psec.

The energy of the system was minimized using the steepest descent method for $20 \mathrm{nsec}$ at $290 \mathrm{~K}$ without any position restraints. Afterwards, one of the equilibrium structures was chosen as the starting structure for further MD simulations at higher (different) temperatures.

In our simulations, in order to characterize the opening/closing of a single base pair, we fixed all other nucleotides except for the two terminal nucleotides ( $A$ and $U$ ) of the RNA hairpin with harmonic constraints, by which the base pairs except the terminal base pair stay paired and could vibrate around their initial positions. So when the terminal base pair is in the closed state as the RMSD is $<2 \AA$, the stacking interaction of the terminal base pair with the nearest-neighbor base pair will not be affected. Each of our simulations lasted $3 \mu \mathrm{sec}$ for each salt concentration and temperature for our analyses on thermodynamic and kinetic parameters. The details of our MD simulations have been described previously in Wang et al. $(2016,2018)$.

\section{ACKNOWLEDGMENTS}

We are grateful to Professors Shi-Jie Chen (University of Missouri) and Xiangyun Qiu (George Washington University) for valuable discussions. This work was partly supported by the National Natural Science Foundation of China under grants 11574234 and 31270761 (to W.B.Z.), and grants 11774272 and 11575128 (to Z.J. T.). The numerical calculations in this work were performed on the supercomputing system in the Super Computing Center of Wuhan University.

Received November 6, 2019; accepted January 11, 2020.

\section{REFERENCES}

Bartel DP. 2004. MicroRNAs: genomics, biogenesis, mechanism, and function. Cell 116: 281-297. doi:10.1016/S0092-8674(04) 00045-5

Bartel DP, Szostak JW. 1993. Isolation of new ribozymes from a large pool of random sequences. Science 261: 1411-1418. doi:10 $.1126 /$ science. 7690155

Bevilacqua PC, Blose JM. 2008. Structures, kinetics, thermodynamics, and biological functions of RNA hairpins. Annu Rev Phys Chem 59: 79-103. doi:10.1146/annurev.physchem.59.032607.093743

Blake RD, Delcourt SG. 1998. Thermal stability of DNA. Nucleic Acids Res 26: 3323-3332. doi:10.1093/nar/26.14.3323

Boerner AR, Kowerko D, Miserachs HG, Schaffer F, Sigel RKO. 2016. Metal ion induced heterogeneity in RNA folding studied by smFRET. Coordin Chem Rev 327-328: 123-142. doi:10.1016/j .ccr.2016.06.002

Bokinsky G, Rueda D, Misra VK, Rhodes MM, Gordus A, Babcock HP, Walter NG, Zhuang X. 2003. Single-molecule transition-state analysis of RNA folding. Proc Natl Acad Sci 100: 9302-9307. doi:10 $.1073 /$ pnas. 1133280100

Brion P, Westhof E. 1997. Hierarchy and dynamics of RNA folding. Annu Rev Biophys Biomol Struct 26: 113-137. doi:10.1146/ annurev.biophys.26.1.113

Bruce JB, Borkovec M, Straub JE. 1988. Classical and modern methods in reaction rate theory. J Phys Chem 92: 3711-3725. doi:10 .1021/j100324a007

Bryngelson JD, Wolynes PG. 1989. Intermediates and barrier crossing in a random energy model (with applications to protein folding). $J$ Phys Chem 93: 6902-6915. doi:10.1021/j100356a007

Bussi G, Donadio D, Parrinello M. 2007. Canonical sampling through velocity rescaling. J Chem Phys 126: 014101-014107. doi:10 $.1063 / 1.2408420$

Casalino L, Magistrato A. 2016. Structural, dynamical and catalytic interplay between $\mathrm{Mg}^{2+}$ ions and RNA. Vices and virtues of atomistic simulations. Inorganica Chim Acta 452: 73-81. doi:10.1016/j.ica .2016.02.011

Chen YL, Pollack L. 2019. The salt dependence of A-form RNA duplexes: structures and implications. J Phys Chem B 123: 9773-9785. doi: 10.1021/acs.jpcb.9b07502

Chen Z, Znosko BM. 2013. Effect of sodium ions on RNA duplex stability. Biochemistry 52: 7477-7485. doi:10.1021/bi4008275 
Chung HS, Eaton WA. 2013. Single-molecule fluorescence probes dynamics of barrier crossing. Nature 502: 685-688. doi:10.1038/ nature12649

Chung HS, Louis JM, Eaton WA. 2009. Experimental determination of upper bound for transition path times in protein folding from single-molecule photon-by-photon trajectories. Proc Natl Acad Sci 106: 11837-11844. doi:10.1073/pnas.0901178106

Darden T, York D, Pedersen L. 1993. Particle mesh Ewald: an $N \cdot \log (N)$ method for Ewald sums in large systems. J Chem Phys 98: 1008910092. doi:10.1063/1.464397

Denesyuk NA, Thirumalai D. 2015. How do metal ions direct ribozyme folding? Nat Chem 7: 793-801. doi:10.1038/nchem.2330

Dokholyan NV, Buldyrev SV, Stanley HE, Shakhnovich El. 2000. Identifying the protein folding nucleus using molecular dynamics. J Mol Biol 296: 1183-1188. doi:10.1006/jmbi.1999.3534

Draper DE. 2008. RNA folding: thermodynamic and molecular descriptions of the roles of ions. Biophys J 95: 5489-5495. doi:10 .1529/biophysj.108.131813

Draper DE, Grilley D, Soto AM. 2005. lons and RNA folding. Annu Rev Biophys Biomol Struct 34: 221-243. doi:10.1146/annurev.biophys .34.040204.144511

Drozdetski AV, Tolokh IS, Pollack L, Baker N, Onufriev AV. 2016. Opposing effects of multivalent ions on the flexibility of DNA and RNA. Phys Rev Lett 117: 028101. doi:10.1103/PhysRevLett .117.028101

Dzyaloshinskii IE, Lifshitz EM, Pitaevskii LP. 1961. General theory of van der Waals' forces. Sov Phys 73: 153-176. doi:10.1070/ PU1961v004n02ABEH003330

Ellington AD, Szostak JW. 1990. In vitro selection of RNA molecules that bind specific ligands. Nature 346: 818-822. doi:10.1038/ $346818 \mathrm{a} 0$

Essmann U, Perera L, Berkowitz ML, Darden T, Lee H, Pedersen LG. 1995. A smooth particle mesh Ewald method. J Chem Phys 103: 8577-8593. doi:10.1063/1.470117

Fischer NM, Polêto MD, Steuer J, Dvd S. 2018. Influence of $\mathrm{Na}^{+}$and $\mathrm{Mg}^{2+}$ ions on RNA structures studied with molecular dynamics simulations. Nucleic Acids Res 46: 4872-4882. doi:10.1093/nar/ gky221

Giudice E, Várnai P, Lavery R. 2003. Base pair opening within B-DNA: free energy pathways for GC and AT pairs from umbrella sampling simulations. Nucleic Acids Res 31: 1434-1443. doi:10.1093/nar/ gkg239

Hänggi P, Talkner P, Borkovec M. 1990. Reaction-rate theory: fifty years after Kramers. Rev Mod Phys 62: 251-341. doi:10.1103/ RevModPhys.62.251

Heilman-Miller SL, Thirumalai D, Woodson SA. 2001. Role of counterion condensation in folding of the Tetrahymena ribozyme. I. Equilibrium stabilization by cations. J Mol Biol 306: 1157-1166. doi:10.1006/jmbi.2001.4437

Hershkovitz E, Tannenbaum E, Howerton SB, Sheth A, Tannenbaum A, Williams LD. 2003. Automated identification of RNA conformational motifs: theory and application to the HM LSU $23 S$ rRNA. Nucleic Acids Res 31: 6249-6257. doi:10.1093/nar/gkg835

Hess B, Bekker H, Berendsen HJC, Fraaije JGEM. 1997. LINCS: a linear constraint solver for molecular simulations. J Comput Chem 18: 1463-1472. doi:10.1002/(SICI)1096-987X(199709)18 :12<1463::AID-JCC4>3.0.CO;2-H

Hess B, Kutzner C, Dvd S, Lindahl E. 2008. GROMACS 4: algorithms for highly efficient, load-balanced, and scalable molecular simulation. J Chem Theory Comput 4: 435-447. doi:10.1021/ ct700301q

Hori N, Denesyuk NA, Thirumalai D. 2019. Ion condensation onto ribozyme is site specific and fold dependent. Biophys $J$ 116: 2400-2410. doi:10.1016/j.bpj.2019.04.037
Hummer G. 2004. From transition paths to transition states and rate coefficients. J Chem Phys 120: 516-523. doi:10.1063/1 .1630572

Humphrey W, Dalke A, Schulten K. 1996. VMD: visual molecular dynamics. J Mol Graph 14: 33-38. doi:10.1016/0263-7855(96)00018-5

Huynen MA, Perelson A, Vieira WA, Stadler PF. 1996. Base pairing probabilities in a complete HIV-1 RNA. J Comput Biol 3: 253274. doi:10.1089/cmb.1996.3.253

Ippolito JA, Steitz TA. 1998. A 1.3-A resolution crystal structure of the HIV-1 trans-activation response region RNA stem reveals a metal ion-dependent bulge conformation. Proc Natl Acad Sci 95: 9819-9824. doi:10.1073/pnas.95.17.9819

Jin L, Shi Y-Z, Feng C-J, Tan Y-L, Tan Z-J. 2018. Modeling structure, stability, and flexibility of double-stranded RNAs in salt solutions. Biophys J 115: 1403-1416. doi:10.1016/j.bpj.2018.08.030

Jin L, Tan Y-L, Wu Y, Wang X, Shi Y-Z, Tan Z-J. 2019. Structure folding of RNA kissing complexes in salt solutions: predicting 3D structure, stability and folding pathway. RNA 25: 1532-1548. doi:10.1261/ rna.071662.119

Jorgensen WL, Chandrasekhar J, Madura JD, Impey RW, Klein ML. 1983. Comparison of simple potential functions for simulating liquid water. J Chem Phys 79: 926-935. doi:10.1063/1.445869

Kim T, Shapiro BA. 2013. The role of salt concentration and magnesium binding in HIV-1 subtype-A and subtype-B kissing loop monomer structures. J Biomol Struct Dyn 31: 495-510. doi:10 $.1080 / 07391102.2012 .706072$

Kirmizialtin S, Pabit SA, Meisburger SP, Pollack L, Elber R. 2012. RNA and its ionic cloud: solution scattering experiments and atomically detailed simulations. Biophys J 102: 819-828. doi:10.1016/j.bpj .2012.01.013

Koculi E, Hyeon C, Thirumalai D, Woodson SA. 2007. Charge density of divalent metal cations determines RNA stability. J Am Chem Soc 129: 2676-2682. doi:10.1021/ja068027r

Kolev SK, Petkov PS, Rangelov MA, Trifonov DV, Milenov TI, Vayssilov GN. 2018. Interaction of $\mathrm{Na}^{+}, \mathrm{K}^{+}, \mathrm{Mg}^{2+}$ and $\mathrm{Ca}^{2+}$ counter cations with RNA. Metallomics 10: 659-678. doi:10.1039/ C8MT00043C

Kuznetsov SV, Ansari A. 2012. A kinetic zipper model with intrachain interactions applied to nucleic acid hairpin folding kinetics. Biophys J 102: 101-111. doi:10.1016/j.bpj.2011.11.4017

Lennard-Jones BJE. 1931. Cohesion. Proc Phys Soc 43: 461-482. doi:10.1088/0959-5309/43/5/301

Li PTX. 2013. Analysis of diffuse $\mathrm{K}^{+}$and $\mathrm{Mg}^{2+}$ ion binding to a twobase-pair kissing complex by single-molecule mechanical unfolding. Biochemistry 52: 4991-5001. doi:10.1021/bi400646x

Lin C, Zhang X, Qiang X, Zhang JS, Tan Z-J. 2019. Apparent repulsion between equally and oppositely charged spherical polyelectrolytes in symmetrical salt solutions. J Chem Phys 151: 114902114908. doi:10.1063/1.5120756

Lipfert J, Doniach S, Das R, Herschlag D. 2014. Understanding nucleic acid-ion interactions. Annu Rev Biochem 83: 813-841. doi:10 .1146/annurev-biochem-060409-092720

Mahoney MW, Jorgensen WL. 2000. A five-site model for liquid water and the reproduction of the density anomaly by rigid, nonpolarizable potential functions. J Chem Phys 112: 8910-8922. doi:10 $.1063 / 1.481505$

Manning GS. 1978. The molecular theory of polyelectrolyte solutions with applications to the electrostatic properties of polynucleotides. Q Rev Biophys 11: 179-246. doi:10.1017/S003358350 0002031

Manning GS. 2001. Counterion condensation on a helical charge lattice. Macromolecules 34: 4650-4655. doi:10.1021/ma010159b

Manning GS. 2007. Counterion condensation on charged spheres, cylinders, and planes. J Phys Chem B 111: 8554-8559. doi:10 $.1021 /$ jp0670844 
Miyamoto S, Kollman PA. 1992. Settle: an analytical version of the SHAKE and RATTLE algorithm for rigid water models. J Comput Chem 13: 952-962. doi:10.1002/jcc.540130805

Nakano SI, Tanino Y, Hirayama H, Sugimoto N. 2016. Thermal stability of RNA structures with bulky cations in mixed aqueous solutions. Biophys J 111: 1350-1360. doi:10.1016/j.bpj.2016.08.031

Nayak RK, Peersen OB, Hall KB, Orden AV. 2012. Millisecond timescale folding and unfolding of DNA hairpins using rapid-mixing stopped-flow kinetics. J Am Chem Soc 134: 2453-2456. doi:10 $.1021 / \mathrm{ja} 208490 \mathrm{w}$

Nudler E, Mironov AS. 2004. The riboswitch control of bacterial metabolism. Trends Biochem Sci 29: 11-17. doi:10.1016/j.tibs.2003 .11 .004

Owczarzy R, You Y, Moreira BG, Manthey JA, Huang L, Behlke MA, Walder JA. 2004. Effects of sodium ions on DNA duplex oligomers: improved predictions of melting temperatures. Biochemistry 43: 3537-3554. doi:10.1021/bi034621r

Owczarzy R, Moreira BG, You Y, Behlke MA, Walder JA. 2008. Predicting stability of DNA duplexes in solutions containing magnesium and monovalent cations. Biochemistry 47: 5336-5353. doi:10.1021/bi702363u

Parrinello M, Rahman A. 1981. Polymorphic transitions in single crystals: a new molecular dynamics method. J Appl Phys 52: 71827190. doi:10.1063/1.328693

Pérez A, Marchán I, Svozil D, Sponer J, Cheatham TE, Laughton CA, Orozco M. 2007. Refinement of the AMBER force field for nucleic acids: improving the description of $\alpha / \gamma$ conformers. Biophys J 92: 3817-3829. doi:10.1529/biophysj.106.097782

Pyle AM. 2002. Metal ions in the structure and function of RNA. J Biol Inorg Chem 7: 679-690. doi:10.1007/s00775-002-0387-6

Qiu X, Parsegian VA, Rau DC. 2010. Divalent counterion-induced condensation of triple-strand DNA. Proc Natl Acad Sci 107: 2148221486. doi:10.1073/pnas.1003374107

Qiu X, Rau DC, Parsegian VA, Fang LT, Knobler CM, Gelbart WM. 2011. Salt-dependent DNA-DNA spacings in intact bacteriophage $\lambda$ reflect relative importance of DNA self-repulsion and bending energies. Phys Rev Lett 106: 028102. doi:10.1103/ PhysRevLett.106.028102

Raper AT, Reed AJ, Suo Z. 2018. Kinetic mechanism of DNA polymerases: contributions of conformational dynamics and a third divalent metal ion. Chem Rev 118: 6000-6025. doi:10.1021/acs .chemrev.7b00685

Rauscher S, Flamm C, Mandl CW, Heinz FX, Stadler PF. 1997. Secondary structure of the $3^{\prime}$-noncoding region of flavivirus genomes: comparative analysis of base pairing probabilities. RNA 3: 779-791.

SantaLucia J. 1998. A unified view of polymer, dumbbell, and oligonucleotide DNA nearest-neighbor thermodynamics. Proc Natl Acad Sci 95: 1460-1465. doi:10.1073/pnas.95.4.1460

SantaLucia J, Hicks D. 2004. The thermodynamics of DNA structural motifs. Annu Rev Biophys Biomol Struct 33: 415-440. doi:10 .1146/annurev.biophys.32.110601.141800

Sarkar K, Nguyen D A, Gruebele M. 2010. Loop and stem dynamics during RNA hairpin folding and unfolding. RNA 16: 2427-2434. doi:10.1261/rna.2253310

Serra MJ, Turner DH. 1995. Predicting thermodynamic properties of RNA. Methods Enzymol 259: 242-261. doi:10.1016/0076-6879 (95)59047-1

Serra MJ, Baird JD, Dale T, Fey BL, Retatagos K, Westhof E. 2002. Effects of magnesium ions on the stabilization of RNA oligomers of defined structures. RNA 8: 307-323. doi:10.1017/S1355838 202024226

Shi Y-Z, Jin L, Feng C-J, Tan Y-L, Tan Z-J. 2018. Predicting 3D structure and stability of RNA pseudoknots in monovalent and divalent ion solutions. PLoS Comput Biol 14: 1-23.
Steinert HS, Rinnenthal J, Schwalbe H. 2012. Individual basepair stability of DNA and RNA studied by NMR-detected solvent exchange. Biophys J 102: 2564-2574. doi:10.1016/j.bpj.2012.03 .074

Sun L-Z, Zhang D, Chen S-J. 2017. Theory and modeling of RNA structure and interactions with metal ions and small molecules. Annu Rev Biophys 46: 227-246. doi:10.1146/annurev-biophys070816-033920

Tan Z-J, Chen S-J. 2006. Nucleic acid helix stability: effects of salt concentration, cation valence and size, and chain length. Biophys J 90: 1175-1190. doi:10.1529/biophysj.105 .070904

Tan Z-J, Chen S-J. 2007. RNA helix stability in mixed $\mathrm{Na}^{+} / \mathrm{Mg}^{2+}$ solution. Biophys J 92: 3615-3632. doi:10.1529/biophysj.106 100388

Tan Z-J, Chen S-J. 2011. Salt contribution to RNA tertiary structure folding stability. Biophys J 101: 176-187. doi:10.1016/j.bpj .2011 .05 .050

Tinoco I, Bustamante C. 1999. How RNA folds. J Mol Biol 293: $271-$ 281. doi:10.1006/jmbi.1999.3001

Vashishtha AK, Wang J, Konigsberg WH. 2016. Different divalent cations alter the kinetics and fidelity of DNA polymerases. J Biol Chem 291: 20869-20875. doi:10.1074/jbc.R116.742494

Wang J, Kollman PA. 2001. Automatic parameterization of force field by systematic search and genetic algorithms. J Comput Chem 22: 1219-1228. doi:10.1002/jcc.1079

Wang J, Xiao Y. 2016. Types and concentrations of metal ions affect local structure and dynamics of RNA. Phys Rev E 94: 040401. doi:10.1103/PhysRevE.94.040401

Wang J, Cieplak P, Kollman PA. 2000. How well does a restrained electrostatic potential (RESP) model perform in calculating conformational energies of organic and biological molecules? J Comput Chem 21: 1049-1074. doi:10.1002/1096-987X(200009) 21:12<1049::AID-JCC3>3.0.CO;2-F

Wang J, Zhao Y, Wang J, Xiao Y. 2015. Computational study of stability of an H-H-type pseudoknot motif. Phys Rev E 92: 062705. doi:10.1103/PhysRevE.92.062705

Wang Y, Gong S, Wang Z, Zhang W. 2016. The thermodynamics and kinetics of a nucleotide base pair. J Chem Phys 144: 115101. doi:10.1063/1.4944067

Wang Y, Wang Z, Wang Y, Liu T, Zhang W. 2018. The nearest neighbor and next nearest neighbor effects on the thermodynamic and kinetic properties of RNA base pair. J Chem Phys 148: 045101. doi:10.1063/1.5013282

Wang F, Sun L-Z, Cai P, Chen S-J, Xu X. 2019a. Kinetic mechanism of RNA helix-terminal basepairing-a kinetic minima network analysis. Biophys J 117: 1674-1683. doi:10.1016/j.bpj.2019.09 .017

Wang Z, Wang Y, Liu T, Wang Y, Zhang W. 2019b. Effects of the PIWI/ MID domain of Argonaute protein on the association of miRNAi's seed base with the target. RNA 25: 620-629. doi:10.1261/rna .069328 .118

Woodson SA. 2005. Metal ions and RNA folding: a highly charged topic with a dynamic future. Curr Opin Chem Biol 9: 104-109. doi:10.1016/j.cbpa.2005.02.004

Xi K, Wang F, Xiong G, Zhang Z, Tan Z-J. 2018. Competitive binding of $\mathrm{Mg}^{2+}$ and $\mathrm{Na}^{+}$ions to nucleic acids: from helices to tertiary structures. Biophys J 114: 1776-1790. doi:10.1016/j.bpj.2018.03 .001

Xia T, SantaLucia J, Burkard ME, Kierzek R, Schroeder SJ, Jiao X, Cox C, Turner DH. 1998. Thermodynamic parameters for an expanded nearest-neighbor model for formation of RNA duplexes with Watson-Crick base pairs. Biochemistry 37: 14719-14735. doi:10.1021/bi9809425 
Xu X, Yu T, Chen SJ. 2016. Understanding the kinetic mechanism of RNA single base pair formation. Proc Natl Acad Sci 113: 116 121. doi:10.1073/pnas. 1517511113

Zgarbová M, Otyepka M, Šponer J, Mládek A, Banáš P, Cheatham TE, Jurečka P. 2011. Refinement of the Cornell et al. nucleic acids force field based on reference quantum chemical calculations of glycosidic torsion profiles. J Chem Theory Comput 7: 28862902. doi:10.1021/ct200162x

Zhang W, Chen S-J. 2002. RNA hairpin-folding kinetics. Proc Natl Acad Sci 99: 1931-1936. doi:10.1073/pnas.032443099
Zhang J, Qin M, Wang W. 2006. Folding mechanism of $\beta$ hairpins studied by replica exchange molecular simulations. Proteins Struct Funct Genet 62: 672-685. doi:10.1002/prot .20813

Zhang Y, Zhang J, Wang W. 2011. Atomistic analysis of pseudoknotted RNA unfolding. J Am Chem Soc 133: 6882-6885. doi:10 $.1021 / j a 1109425$

Zhao P, Zhang W, Chen S-J. 2010. Predicting secondary structural folding kinetics for nucleic acids. Biophys J 98: 1617-1625. doi:10.1016/j.bpj.2009.12.4319 

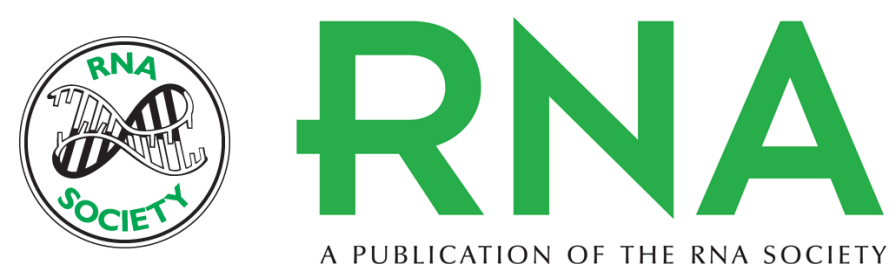

A PUBLICATION OF THE RNA SOCIETY

\section{Salt effect on thermodynamics and kinetics of a single RNA base pair}

Yujie Wang, Taigang Liu, Ting Yu, et al.

RNA 2020 26: 470-480 originally published online January 27, 2020

Access the most recent version at doi:10.1261/rna.073882.119

References This article cites 95 articles, 14 of which can be accessed free at: http://rnajournal.cshlp.org/content/26/4/470.full.html\#ref-list-1

Creative This article is distributed exclusively by the RNA Society for the first 12 months after the Commons full-issue publication date (see http://rnajournal.cshlp.org/site/misc/terms.xhtml). After 12 License months, it is available under a Creative Commons License (Attribution-NonCommercial 4.0 International), as described at http://creativecommons.org/licenses/by-nc/4.0/.

Email Alerting Receive free email alerts when new articles cite this article - sign up in the box at the Service top right corner of the article or click here.

To subscribe to $R N A$ go to:

http://rnajournal.cshlp.org/subscriptions

(C) 2020 Wang et al.; Published by Cold Spring Harbor Laboratory Press for the RNA Society 